\title{
Research on Chemical Properties of Pyrolysis Products of Gentamicin Residue
}

\author{
Jia Meng-meng, Li Yi-fei ${ }^{*}$, Feng Li-hui, Wang Zhi-qiang, Ma Si-Lu, Yang Jian, Hu Jia-shuo \\ School of Energy and Environmental Engineering, University of Science and Technology Beijing, Beijing, China
}

\section{Email address:}

ustbxiaojiameng@163.com (Jia Meng-meng), lyf-33053085@163.com (Li Yi-fei)

${ }^{*}$ Corresponding author

\section{To cite this article:}

Jia Meng-meng, Li Yi-fei, Feng Li-hui, Wang Zhi-qiang, Ma Si-Lu, Yang Jian, Hu Jia-shuo. Research on Chemical Properties of Pyrolysis Products of Gentamicin Residue. Science Discovery. Vol. 5, No. 6, 2017, pp. 457-462. doi: 10.11648/j.sd.20170506.21

Received: October 1, 2017; Accepted: October 8, 2017; Published: November 21, 2017

\begin{abstract}
This paper selected gentamicin ferment residue in the tube furnace reactor for rapid pyrolysis experiments, the ferment residue and pyrolysis products (coke, tar) were analyzed by XPS, FTIR and GC-MS. FTIR analysis showed that the ferment residue and pyrolysis products mainly contain saturated alkanes and proteins, sugars and lipids. XPS results show that the pyrolytic coke is mainly C-C chemical bond, its content increased significantly from $55.07 \%$ to $72.27 \%$, while the C-N bond from $18.21 \%$ to $5.8 \%$. The chemical constituents of the pyrolysis oil were separated and identified by GC-MS. The identified materials could be divided into: aromatic hydrocarbons, phenol and its derivatives, esters, ketones, acids, amines, nitriles and some heterocyclic compounds; The effect of the pyrolysis temperature on the main components of pyrolysis oil was studied. It was found that the effect of temperature on the composition of the components was not significant, but the relative content of the components was significant. At the same time, the nitrogen-containing compounds in the pyrolysis oil are pyrrole, nitriles and indole, and the content is $9.93 \%-29.17 \%$.
\end{abstract}

Keywords: Gentamicin Ferment Residue, Pyrolysis, XPS, FTIR, GC-MS

\section{庆大霉素菌渣热解产物化学特性研究}

贾蒙蒙, 李益飞*, 冯丽慧, 王志强, 马思路, 杨健, 胡家硕

能源与环境工程学院, 北京科技大学, 北京, 中国

邮箱

ustbxiaojiameng@163.com (贾蒙蒙), lyf4888@qq.com（李益飞）

摘要: 选取庆大霉素菌渣在管式炉反应器中进行快速热解实验, 利用XPS、FTIR和GC-MS对菌渣以及热解产物 (焦炭, 焦油）进行分析。FTIR分析显示，可知菌渣及热解产物中主要含有饱和烷烃及蛋白质、糖类和脂类化合物。XPS结果 表明, 热解焦炭中主要为C-C化学键, 其含量由 $55.07 \%$ 大幅升至 $72.27 \%$, 而C-N键由 $18.21 \%$ 降至 $5.8 \%$; 用GC-MS法分 离和鉴定热解油的化学成分, 检索得到的物质可分为: 芳烃类化合物、苯酚及其衍生物、酯、酮、酸、胺、腈以及一 些杂环化合物; 对比热解温度对热解油主要组分的影响, 发现温度对组分的组成影响不大, 而对各组分物质的相对含 量影响较大; 同时也得到热解油中含氮化合物主要为吡咯、腈类、吲哚等，其含量为9.93\%-29.17\%。

关键词: 庆大霉素菌渣, 热解, XPS, FTIR, GC-MS 


\section{1. 引言}

中国是抗生素类药物生产大国, 2013年产生的抗生素 菌渣类废弃物已达到 1400 万吨 [1]。抗生素菌渣是抗生素发 酵工艺产生的有机废物，由菌丝体、剩余培养基成分、中 间代谢产物及少量的残留抗生素等[2]组成包含大量蛋白 质、脂肪、多糖等物质 [3,4]。由于抗生素菌渣的含水率高、 产生量大且有异味, 制约了其按照填埋或焚烧的方式进行 处理 [5]。因此迫切需要针对菌渣特点, 开发合理、可行、 安全的处理技术, 为实现无害化、减量化、资源化提供技 术保证。

目前国内外学者对菌渣处理技术进行了许多了研究, 如庈氧发酵[6], 处理后用作蛋白饲料或添加剂[7]、提取 纯化核糖核酸 [8]等, 但存在成本高、处理效率低、二次污 染等问题。此外, 周保华等[9]用实验方法对青霉素、土霉 素菌渣成分进行分析, 表明青霹素、土霉素菌渣中有机物 含量较高, 而重金属、无机成分和多环芳烃含量较少。贡 丽鹏等[10]以制药厂土霉素、青霉素菌渣为对象的研究结 果表明, 菌渣中C、O元素含量较高, 以上性质符合热解 气化技术对原材料的要求。

因此有必要开展对抗生素菌渣热解处理技术的研究, 热解技术不仅可以使菌渣减容、减量, 而且可以实现菌渣 的资源化利用, 但直到目前, 热解处理技术仍存在许多问 题, 如热解机理有待完善, 热解产物的化学特性有待深入 探索。本文针对以上问题, 以抗生素菌渣为研究对象, 研 究不同热解条件下热解产物的性质以及官能团分布随热 解温度的变化规律, 为深入了解抗生素菌渣的热解机理、 热解产物的资源化利用提供参考, 为合理开发和利用抗生 素菌渣资源提供理论依据。

\section{2. 分析指标与方法}

实验分析指标与分析方法见表3.

\section{2. 材料与方法}

\section{1. 样品处理及实验过程}

本试验所用的庆大霉素菌渣来自于石家庄某抗生素 制药集团, 首先将菌渣放入 $105^{\circ} \mathrm{C}$ 烘箱内干燥 $24 \mathrm{~h}$, 将干燥 后的菌渣研磨篮分, 使其粒径小于 $1 \mathrm{~mm}$, 再将样品进行充 分混合, 以保证原料的均匀性; 菌渣元素和工业分析以及 主要有机质含量分析结果见表1、2.试验采用管式炉热解 反应器热解菌渣, 热解气氛为高纯度 $\mathrm{N}_{2}(99.999 \%)$ 。先将 氮气瓶打开, 流量设定为 $500 \mathrm{ml} / \mathrm{min}$, 排尽系统内的空气。 然后将盛放干燥原料(每次约 $16 \mathrm{~g}$ ) 的管式炉热解器放置在 反应器左侧非加热区, 当右侧热解区达到设定温度后(设 定为 $400 、 500 、 600 、 700^{\circ} \mathrm{C}$, 升温速率 $\left.10^{\circ} \mathrm{C} / \mathrm{min}\right)$, 将容 器推入热解区域。每次实验恒温热解 $180 \mathrm{~min}$, 通过冷凝装 置收集液体产物, 进行称量, 待反应完全 (此时由FTIR检测 到的气体组分的曲线趋于水平) 后, 将反应器在氮气保护 下降至室温, 与反应前对比获得固体产物的质量。所有的 实验重复 3 次以确保结果的再现性, 平均实验误差为 $\pm 5 \%$ 。

表1 庆大霉素菌渣元素分析。

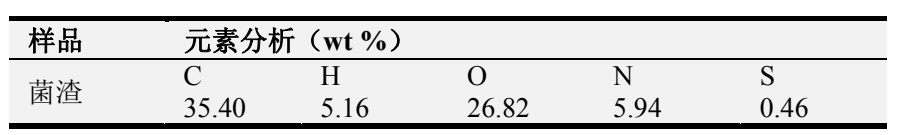

表2 庆大霉素菌渣工业分析及有机质含量分析。

\begin{tabular}{lllllll}
\hline 样品 & \multicolumn{1}{c}{ 工业分析 $(\mathbf{w t} \%)$} & & & \multicolumn{2}{c}{ 有机质含量 $(\%)$} \\
\hline & $\mathrm{M}$ & $\mathrm{A}$ & $\mathrm{V}$ & $\mathrm{FC}$ & protein & fat \\
菌渣 & 4.80 & 21.42 & 63.74 & 10.04 & 48.6 & 6.28 \\
\hline
\end{tabular}

表3 检测指标与分析方法。

\begin{tabular}{|c|c|}
\hline 检测指标 & 分析方法与仪器 \\
\hline \multirow{3}{*}{ 菌渣元素分析 $(\mathrm{C} 、 \mathrm{H} 、 \mathrm{O} 、 \mathrm{~N} 、 \mathrm{~S})$} & GB/T28732-2012《固体生物质燃料全硫测定方法》 \\
\hline & GB/T28734-2012《固体生物质燃料中碳氢测定方法》 \\
\hline & GB/T30728-2014《固体生物质燃料中氮的测定方法》 \\
\hline 菌渣工业分析（M、A、V、FC） & GB/T28731-2012《固体生物质燃料工业分析方法》 \\
\hline 菌渣有机质分析（蛋白质、脂肪） & 凯氏定氮法测定、索氏提取法测定 \\
\hline 热解焦炭化学键C1s & 热解炭-X射线光电子能谱, 岛津Kratos AXIS ULTRADLD \\
\hline 热解焦炭和焦油有机官能团 & 傅里叶变换红外光谱仪, 美国Thermo Fisher NicoletiS50 \\
\hline 热解焦油化学组成成分 & 采用气-质色谱联用分析仪(GC-MS) Agilent Technologies 7693 \\
\hline
\end{tabular}

注: 元素分析中利用差减法得到 $\mathrm{O}$ 的含量, 计算公式: Oad=100-Mad-Aad-Cad-Had-Nad-Sad;工业分析中, M: 水分; A: 灰分; V: 挥发分; FC: 固 定碳

\section{3. 结果与讨论}

\section{1 . 焦炭产物的碳官能团分析}

\section{1. 1 . 傅里叶红外光谱分析}

在菌渣热解炭的红外光谱分析中, 红外吸收带的位置 及其相对强度是热解炭官能团组成、键合情况及其振动性 质的反映。庆大霉素菌渣热解炭红外光谱图如图1所示。
为了表达清晰简洁, 在下文中不同热解温度下的炭产 物表达为“C + 温度”, 如 $\mathrm{C} 400$ 为 $400^{\circ} \mathrm{C}$ 的炭产物。

从图中可以看出, 不同热解温度下, 原样与 4 种不同 炭化温度的热解炭的特征吸收峰几乎一样, 仅有微弱差别, 这说明他们表面的化学基团有比较高的一致性。

原样及热解炭在 $3600 \mathrm{~cm}^{-1}$ 附近出现糖类或苯酚类 $\mathrm{C}-\mathrm{OH}$ 的 $\mathrm{OH}$ 伸缩振动吸收峰; 同时, 原样和 $\mathrm{C} 400$ 在 $3440 \mathrm{~cm}^{-1}$ 左右出现 $\mathrm{N}-\mathrm{H}$ 伸缩振动吸收峰, 来自于蛋白质的 
分解; 在原样中 $2098 \mathrm{~cm}^{-1}$ 处, 为 $\mathrm{C} \equiv \mathrm{C}$ 伸缩振动峰, 随温度 加热升高, 化学键断裂分解; $2935 \mathrm{~cm}^{-1}$ 处, 为环烷或脂肪 族中的甲基 $\mathrm{CH}_{3}$ 反对称伸缩振动, 随着碳化温度升高, 吸 收峰逐渐减弱至基本消失; 在快速 $2280 \mathrm{~cm}^{-1}$ 左右, 为脂肪 族氰化物 (如氰乙酸) 的 $\mathrm{C} \equiv \mathrm{N}$ 伸缩振动; $1665 \mathrm{~cm}^{-1}-1695 \mathrm{~cm}^{-1}$ 之间, 为芳香醛、芳香酮及酰胺羰基的 $\mathrm{C}=\mathrm{O}$ 伸缩振动, 且 随着温度升高, 峰呈先减弱后增强的趋势, 含氧的羰、羧 基团易于断裂, 较长的脂肪族支链或侧链逐渐裂解, 除非 演化程度特别高, 芳核上连结的支链的连结点数目一般不 会因支链的裂解而有大的减少。随着脂肪族支链的不断裂 解, 分子结构中芳构化程度不断增加[11]; 位于 $1520 \mathrm{~cm}^{-1}$ 处的吸收峰, 为酰胺键的 N-H弯曲振动和 $\mathrm{C}-\mathrm{N}$ 伸缩振动; 原样 $1045 \mathrm{~cm}^{-1}$ 以及 $1037 \mathrm{~cm}^{-1}(\mathrm{C} 400) 、 1045 \mathrm{~cm}^{-1}(\mathrm{C} 500)$ 、 $1030 \mathrm{~cm}^{-1}(\mathrm{C} 600) 、 1041 \mathrm{~cm}^{-1}(\mathrm{C} 700)$ 处的吸收峰为 (C-O-C) 脂环醚键的反对称伸缩振动; 原样 $765 \mathrm{~cm}^{-1} 、 752 \mathrm{~cm}^{-1}(\mathrm{C} 400)$ 、 $740 \mathrm{~cm}^{-1}(\mathrm{C} 600) 、 730 \mathrm{~cm}^{-1}(\mathrm{C} 700)$ 为苯环中 $=\mathrm{C}-\mathrm{H}$ 面外 弯曲振动吸收峰。

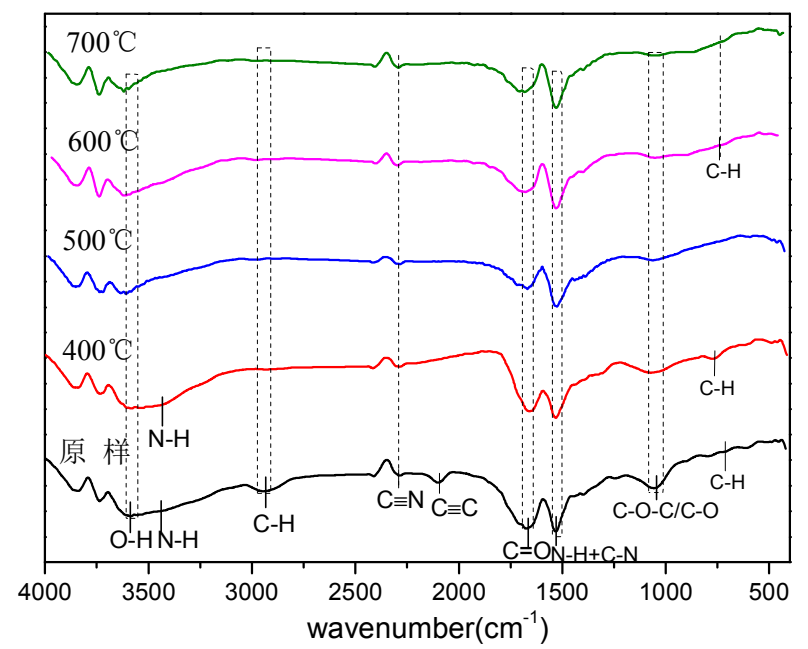

图1 庆大霰素菌渣和各热解温度下形成的热解炭的FTIR谱图。

表4 庆大霉素菌渣热解炭中各官能团的FTIR吸收峰归属。

\begin{tabular}{ll}
\hline 峰位 $\left(\mathbf{c m}^{-1}\right)$ & 吸收峰的振动形式及对应结构 \\
\hline 3600 & 糖类或苯酚类 $\mathrm{C}-\mathrm{OH}$ 的 $\mathrm{OH}$ 伸缩振动 \\
3440 & 胺、酰胺中的 $\mathrm{N}-\mathrm{H}$ 伸缩振动吸收 \\
2935 & 环烷或脂肪族中的甲基 $\mathrm{CH}_{3}$ 反对称伸缩振动 \\
2280 & 脂肪族氰化物 (如氧乙酸) 的 $\mathrm{C} \equiv \mathrm{N}$ 伸缩振动 \\
2100 & 脂肪族中的 $\mathrm{C} \equiv \mathrm{C}$ 伸缩振动 \\
$1665-1695$ & 芳香醛、芳香酮及酰胺羰基的 $\mathrm{C}=\mathrm{O}$ 伸缩振动 \\
1520 & 酰胺键的 $\mathrm{N}-\mathrm{H}$ 弯曲振动和 $\mathrm{C}-\mathrm{N}$ 伸缩振动 \\
$1030-1045$ & $\mathrm{C}-\mathrm{O}-\mathrm{C}$ 脂环醚键的反对称伸缩振动 \\
$730-765$ & 苯环中 $=\mathrm{C}-\mathrm{H}$ 面外弯曲振动 \\
\hline
\end{tabular}

由以上分析, 对比菌渣原样与热解炭的官能团, 可知 菌渣及热解炭中主要含有饱和烷烃及蛋白质、糖类和脂类 化合物, 这些成分中有大量含羧基、羟基和羰基的物质。 元素分析结果显示菌渣中还含有少量 $N$ 和 $S$, 这两种元素 主要作为取代基而存在。另外, 在菌渣及热解炭中均出现 气态 $\mathrm{H}_{2} \mathrm{O}$ 振-转光谱 $\left(3679 \mathrm{~cm}^{-1} 、 3716 \mathrm{~cm}^{-1}\right.$ 和 $3750 \mathrm{~cm}^{-1}$ 左右 $)$, 这可能是由于背景干扰或炭吸附了空气中的气态 $\mathrm{H}_{2} \mathrm{O}$ 所 致[12]。

\section{1.2. XPS-C1s分析}

对庆大霉素菌渣及其热解焦炭的表面处于化学结合 状态 $\mathrm{C} 1 \mathrm{~s}$ 进行分峰处理, 根据C-C、C-N、C-O、C=O、O-C=O 峰的面积值变化, 可以推断其表面各个官能团的含量的变 化规律; 其各官能团的比例变化和表面化学结构特性如图 2、3所示, 其 $\mathrm{C} 1 \mathrm{~s}$ 谱图可以拟合 5 个峰,按照键能从小到大 的顺序排列, 对应的官能团依次为碳碳键 $(\mathrm{C}-\mathrm{C})$ 、碳氮键 $(\mathrm{C}-\mathrm{N})$ 、羟基或醚基 $(\mathrm{C}-\mathrm{OH} / \mathrm{C}-\mathrm{O})$ 、羰基 $(\mathrm{C}=\mathrm{O}$ 或 $\mathrm{O}-\mathrm{C}-\mathrm{O})$ 、(羧 基或酯类, $\mathrm{O}-\mathrm{C}=\mathrm{O})$, 分别记为 $\mathrm{C}_{1} 、 \mathrm{C}_{2} 、 \mathrm{C}_{3} 、 \mathrm{C}_{4} 、 \mathrm{C}_{5}$, 相对 应的结合能分别为 $284.8 \mathrm{eV} 、(285.3 \pm 0.2) \mathrm{eV} 、(286.3 \pm 0.2) \mathrm{eV}$ 、 $(287.5 \pm 0.2) \mathrm{eV}$ 和 $(289 \pm 0.2) \mathrm{eV}[3,13,14]$ 。
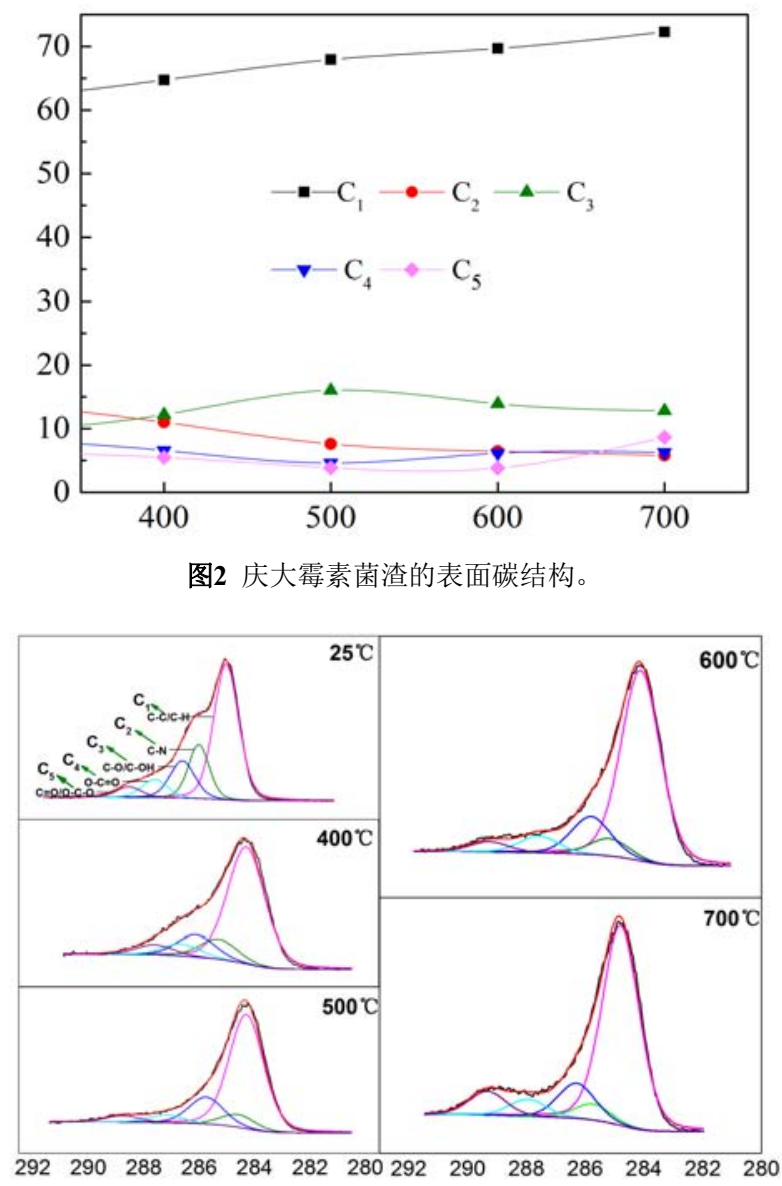

图3 庆大需素菌渣的XPS(C1s) 图谱及分峰结果。

由图2、3可以看出, 当温度从 $25^{\circ} \mathrm{C}$ 升高至 $700^{\circ} \mathrm{C}$ 变化 时, 官能团比例发生明显变化, $\mathrm{C}_{1}$ 比例呈线性上升趋势, 由 $55.07 \%$ 大幅升至 $72.27 \%$, 为碳元素主要存在形式, 表明 庆大霉素菌渣热解过程中发生芳构化反应, 促进了芳香组 分的生成, 进而导致C-C键比例增加[15]; 庆大霉素菌渣 中的含氮组分, 如胺类、蛋白质氨基等在热解过程中发生 分解, 并释放出 $\mathrm{NH}_{3} 、 \mathrm{HCN} 、 \mathrm{NOx}$ 等气体 [16]。因此 $\mathrm{C}_{2}$ 比 例呈降低趋势, 由 $18.21 \%$ 降至 $5.8 \%$, 菌渣的主要成分是蛋 白质、脂类和糖类，这些成分中有大量含羧基、羟基和羰 基的物质, 由图中看出这些含氧官能团的比例之和变化不 明显。 $\mathrm{C}_{3}$ 比例呈现先降低后升高再降低的趋势, 这可能是 由于菌渣中的脂肪族 $\mathrm{C}-\mathrm{O}$ 官能团热解温度较低时 $\left(400^{\circ} \mathrm{C}\right)$ 
发生分解, 而在高温下 $\left(500^{\circ} \mathrm{C}\right)$ 又有一定量的芳香族 $\mathrm{C}-\mathrm{O}$ 官 能团生成, 研究指出 $\mathrm{C}-\mathrm{O}$ 键和 $\mathrm{C}=\mathrm{O}$ 键较弱, 随着温度的升 高逐渐减少 [17]。 $\mathrm{C}_{4}$ 比例先降低后升高（同红外分析结果 一致）, 在 $500^{\circ} \mathrm{C}$ 比例降为 $4.61 \%$, 在温度 $25^{\circ} \mathrm{C}-500^{\circ} \mathrm{C}$ 过程 中, 由于糖类热解导致下降, 随温度继续升高, 菌渣发生 芳构化反应, 促进了芳香组分（芳香醛、芳香酮及芳香族 酰胺）的生成, 进而导致比例升高。 $\mathrm{C}_{5}$ 比例在 $25^{\circ} \mathrm{C}-600^{\circ} \mathrm{C}$ 变化不明显, 当温度为 $700^{\circ} \mathrm{C}$ 时比例增加到 $8.66 \%$, 可能是 芳香酸或芳香酯的生成。

\section{2. 焦油产物的官能团和组分分析}

\subsection{1. 热解油官能团分析一FTIR}

由图4可以看出, 不同热解温度下, 4 种不同热解温度 的热解油的特征吸收峰出现位置几乎一样, 仅峰的强弱有 所差别。这均表明菌渣热解, 性质发生了变化以及热解温 度对菌渣热解产物的含量有明显的影响结果。菌渣热解油 在峰位 $3240 \mathrm{~cm}^{-1}$ 出现宽而强的吸收峰, 为 $\mathrm{O}-\mathrm{H}$ 和 N-H伸缩 振动产生的吸收峰; 在 $2933 \mathrm{~cm}^{-1}$ 和 $2852 \mathrm{~cm}^{-1}$ 处的吸收峰分 别为饱和烷烃 (烷基无序排列) 中亚甲基 $\mathrm{CH}_{2}$ 的对称、反 对称伸缩振动峰; 在峰位 $2212 \mathrm{~cm}^{-1}$ 处, 为不饱和氰的 $\mathrm{C} \equiv \mathrm{N}$ 伸缩振动峰; 在峰位 $2044 \mathrm{~cm}^{-1}$ 处为炔类 $\mathrm{C} \equiv \mathrm{C}$ 伸缩振动峰; 在峰位 $1716 \mathrm{~cm}^{-1}$ 处, 为羟基吸收峰, 为酚、醇、羧酸中的 $\mathrm{O}-\mathrm{H}$ 伸缩振动; 在峰位 $1550 \mathrm{~cm}^{-1}$ 处, 为 $\mathrm{N}-\mathrm{H}$ 胺键面内弯曲 振动; 在峰位 $1460 \mathrm{~cm}^{-1}$ 处峰较弱, 为甲基和亚甲基 $\mathrm{C}-\mathrm{H}$ 面 内弯曲振动; 在峰位 $1385 \mathrm{~cm}^{-1}$ 处的吸收峰, 为 $\mathrm{CH}_{3}$ 甲基的 对称变角振动; 在峰位 $1190 \mathrm{~cm}^{-1}$ 为 C-O-C脂肪醚键伸缩振 动峰; 在峰位 $1296 \mathrm{~cm}^{-1}$ 处出现的吸收峰, 为 $\mathrm{C}-\mathrm{N}$ 胺键的伸 缩振动; 在峰位 $744 \mathrm{~cm}^{-1}$ 附近为其他元素取代 $\mathrm{H}$ 的 $\mathrm{C}$-伸缩振 动峰 $[18]$ 。

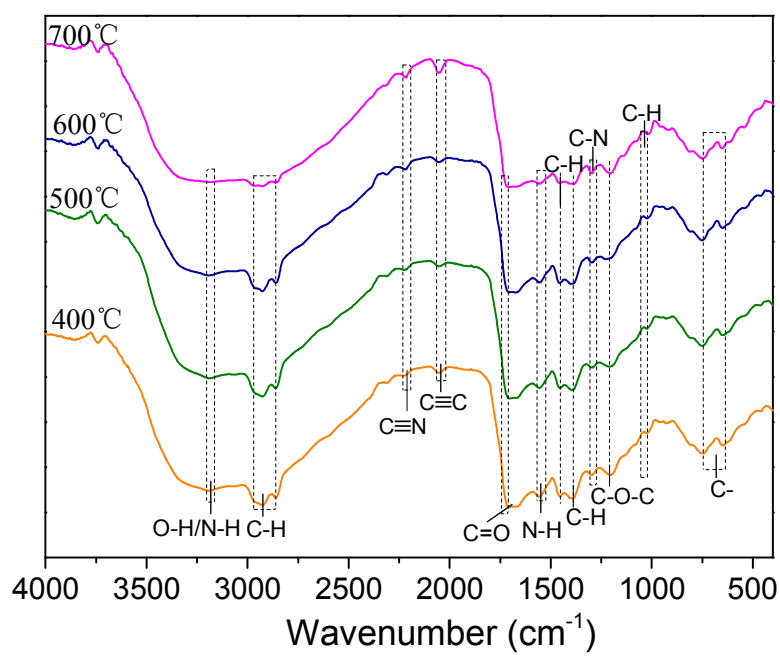

图4 庆大霉素菌渣各热解温度下形成的热解油的FTIR谱图。

\subsection{2. 热解油组成成分分析-GC-MS}

从图5可以看出, 四种热解焦油的GC-MS总离子流图 的主要峰分离效果较好且有明显区别, 主要体现在出峰强 度上, 且出峰强度随温度升高呈变强趋势; 不同热解温度 下, 菌渣热解油均在 $7 \mathrm{~min} 、 15 \mathrm{~min} 、 23 \mathrm{~min} 、 37 \mathrm{~min} 、 60 \mathrm{~min}$ 、 $63 \mathrm{~min}$ 左右出现优势峰; 通过将总离子流图中的各峰与标 准图库比对, 可检测到且匹配度比较高的物质主要含有 8 类物质, 共37余种成分, 按其类别分类主要包括: 芳烃类 化合物、苯酚及其衍生物、酯、酮、酸、胺、腈以及一些 杂环化合物, 如图6所示。

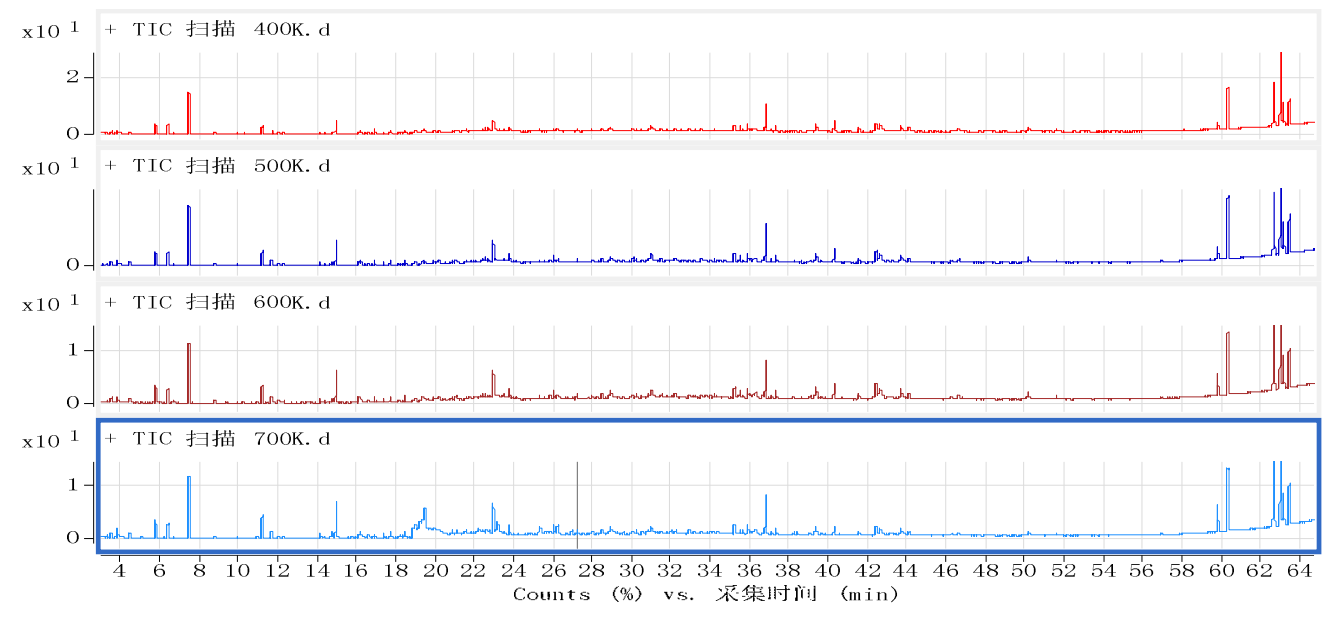

图5 庆大霰素菌渣四种热解温度下焦油产物的GC-MS总离子流图。

菌渣热解焦油中的化学成分十分复杂, 芳烃类物质占 热解油主要组成, 主要为一环芳烃; Maggi等[19]认为这 些芳香族化合物不是生物质快速热裂解的直接产物, 可能 是在存放过程中发生二次反应而产生的, 二次反应的类型 主要有酸和醇的反应、醛和水的反应、醛和醇的反应、醛 和酚在酸性条件下的反应; 含 $\mathrm{O} 、 \mathrm{~N}$ 等杂原子物质也在热 解油成分中占有很大比例, 其中含氧物质含量最高, 其组
成包括酮类化合物, 酚类化合物, 还有部分有机酸类, 这 些极性化合物的存在使得热解油具有亲水性, 其含水量较 高不易去除导致热解油的可燃性降低, 因此需要对热解油 进行相应的改性处理来降低氧的含量, 如催化加氢等[20]; 而其杂环化合物又主要包括呋喃、吡啶、吲哚和哌嗪化合 物, 均属于含氮化合物, 而又从热解油成分中得到含氮化 合物主要为吡咯、吡啶、酰胺、胺类、腈类、吲哚、哌嗪、 
酮肜, 且含氮化合物含量为 $(9.93-29.17 \%)$, 且菌渣热解 油中化合物含量按含 $\mathrm{C}$ 原子个数统计, 可得到含 $\mathrm{C}_{1}-\mathrm{C}_{12}$ 和 $\mathrm{C}_{13}-\mathrm{C}_{18}$ 化合物含量较多, 分别为8.1-18.96和9.54-24.49, 与 上述GC-MS总离子流图中, 出峰强度随温度减弱相对应, 出峰强度决定了焦油组成成分的含量。从而可以得出, 热 解温度对热解油的组成影响不大, 而对成分含量有一定影 响。

由于抗生素菌渣的主要成分是蛋白质, 糖类和脂肪化 合物, 在热解过程中, 原料发生脱水, 裂解等反应, 苯酚 类化合物可能来自于烃类的热解 [21]; 呋喃可能源于糖类 中纤维素和半纤维素的分解[22]。焦油中存在的含氮杂环 化合物, 如吡啶、呋喃等, 是由于原料中的蛋白质热裂解 生成了焦油中的胺类化合物, 随着温度的升高焦油中的胺 态-N裂解生成含氮杂环化合物 [23]。所以菌渣热解液体产 物热解油的各种化合物主要是由于菌渣中三组分热裂解 的化合物产物进一步反应而得。


图6 庆大霉素热解油的主要化学成分分类。

\section{4. 结论}

（1）FTIR分析：可知菌渣及热解产物中主要含有饱 和烷烃及蛋白质、糖类和脂类化合物, 这些成分中有大量 含羧基、羟基和羰基的物质。不同热解温度下出现的特征 吸收峰位置一样, 仅在峰的强弱上有差别, 这均表明菌渣 热解, 性质发生了变化以及热解温度对菌渣热解产物的含 量有明显的影响结果。

（2）XPS分析结果表明, 菌渣热解焦炭中, 表面 $\mathrm{C}$ 主 要以（C-C、C-N、C-O、 $\mathrm{C}=\mathrm{O} 、 \mathrm{O}-\mathrm{C}=\mathrm{O} ）$ 碳的形式存在, $\mathrm{C}-\mathrm{C}$ 键是其主要存在形式, 其含量由 $55.07 \%$ 大幅升至 $72.27 \%$ 。
（3）GC-MS对热解油组成进行分析, 得出：菌渣热 解油主要含有 8 种物质, 按其类别分类主要包括: 芳烃类 化合物、苯酚及其衍生物、酯、酮、酸、胺、腈以及一些 杂环化合物; 且含氮化合物主要为吡咯、吡啶、酰胺、胺 类、腈类、吲哚、哌嗪、酮肟, 含量为 $9.93 \%-29.17 \%$, 这 对以后关于研究温度对生物质热解时氮的迁移转化规律 提供了很好的理论参考。另外热解油中含 $\mathrm{C}_{13}-\mathrm{C}_{18}$ 和 $\mathrm{C}_{1}-\mathrm{C}_{12}$ 化合物含量较多, 分别为 $8.1 \%-18.96 \%$ 和 $9.54 \%-24.49 \%$, 所以可通过进一步的加工制取优质燃料油及化工产品, 对 生物质热解油的基本特性以及有效能源的充分利用具有 积极的参考意义。

\section{参考文献}

[1] 王攀, 于宏兵, 薛旭方, 等. 废弃植物中药渣的热解特性及 动力学研究 [J]. 环境工程学报. 2010(09):2115-2119。

[2] Li C, Zhang G, Zhang Z, et al. Hydrothermal pretreatment for biogas production from anaerobic digestion of antibiotic mycelial residue $[\mathrm{J}]$. Chemical Engineering Journal, 2015, 279:530-537.

[3] Ma D, Zhang G, Zhao P, et al. Hydrothermal treatment of antibiotic mycelial dreg: More understanding from fuel characteristics [J]. Chemical Engineering Journal, 2015, 273:147-155.

[4] 韩洪军, 牟晋铭, 马文成, 等.微波辐射对青霉素菌渣破壁 效果的影响[J].化工学报. 2013(10): 3812-3817。

[5] 杨帅, 张兆玲, 孟剑峰, 等. 循环流化床中菌渣热解气化特 性的研究 [J]. 高校化学工程学报. 2015(04):997-1002。

[6] Baer D R, Engelhard M H. XPS analysis of nanostructured materials and biological surfaces [J]. Journal Of Electron Spectroscopy And Related Phenomena, 2010, 178:.415-432.

[7] 可春昌. 土霉素菌体蛋白饲料的开发利用 [J]. 饲料工 业. 1997,18(8):23-24。

[8] 张红娟.抗生素菌渣堆肥化处理研究[D].郑州大学,2010。

[9] 周保华, 高勤, 王洪华，等.青霉素、土霉素菌渣研究利用 现状及特性分析 [J]. 河北工业科技. 2011(05):291-294。

[10] 贡丽鹏, 郭斌, 任爱玲, 等. 抗生素菌渣理化特性 [J]. 河北 科技大学学报. 2012(02):190-196。

[11] 李庆钊, 林柏泉, 赵长遂, 等. 基于傅里叶红外光谱的高温 煤焦表面化学结构特性分析 [J]. 中国电机工程学 报. 2011(32):46-52。

[12] 唐利兰. 城市生活垃圾热解产物性质及元素分布规律研究 [D]. 重庆大学，2015。

[13] Liao B Q, Lin H J, Langevin S P, et al. Effects of temperature and dissolved oxygen on sludge properties and their role in bioflocculation and settling [J]. Water Research, 2011, 45:509-520. 
[14] Pilawa B, Wi Ckowski A B, Pietrzak R, et al. Oxidation of demineralized coal and coal free of pyrite examined by EPR spectroscopy [J]. Fuel, 2002, 81:1925-1931.

[15] 洪晨, 王志强, 邢奕, 等. 热解温度对土霉素菌渣焦炭化学 性质的影响 [J]. 中国环境科学. 2017,37(3):1058-1065。

[16] Zhu X, Yang S, Wang L, et al. Tracking the conversion of nitrogen during pyrolysis of antibiotic mycelial fermentation residues using XPS and TG-FTIR-MS technology [J]. Environmental Pollution, 2016, 211: 20-27.

[17] 胡强, 陈应泉, 杨海平, 等. 温度对烟杆热解炭、气、油联 产特性的影响 [J]. 中国电机工程学报. 2013(26):54-59。

[18] 吴汉銪, 刘荣厚, 邓春健. 木屑快速热裂解生物油特性及其 红外光谱分析 [J]. 农业工程学报. 2009(06):219-223。
[19] Maggi R, Delmon B. Comparison between 'slow' and 'flash' pyrolysis oils from biomass [J]. Fuel, 1994, 73: 671-677.

[20] 杨巧利, 刘一真, 张瑞芹. GC-MS 分析生物质热解油的研 究 [J]. 郑州大学学报(理学版). 2007(03):140-144。

[21] Chen Y, Yang H, Wang X, et al. Biomass-based pyrolytic polygeneration system on cotton stalk pyrolysis: Influence of temperature [J]. Bioresource Technology, 2012, 107: 411-418.

[22] Yu H, Zhang Z, Li Z, et al. Characteristics of tar formation during cellulose, hemicellulose and lignin gasification $[\mathrm{J}]$. Fuel, 2014, 118: 250-256.

[23] 张军. 微波热解污水污泥过程中氮转化途径及调控策略[D]. 哈尔滨工业大学，2013。 